\title{
ESTIMATION TO EFFICIENCY OF THE MULTIFUNCTION METHOD OF ENDOMETRIOSIS OVARIES TREATMENT
}

D0I: 10.36740/WLek202005106

\author{
Iryna M. Nikitina, Volodymyr I. Boiko, Svitlana A. Smiian, Tetiana V. Babar, Natalia V. Kalashnyk, Alesia V. Boiko, \\ Natalia A. Ikonopystseva, Maryna A. Bolotna \\ SUMY STATE UNIVERSITY, SUMY, UKRAINE
}

\begin{abstract}
The aim: The aim of the study was to improve the results of treatment of patients with endometriosis by using a combination method of therapy. Materials and methods: For two years, 136 women of reproductive age who underwent laparoscopic surgeries for ovarian endometriosis were monitored: Group I ( $\mathrm{n}=24$ ) did not receive any hormonal treatment in the perioperative period; Group II $(n=32)$ - received gonadotropin-releasing hormone agonists within 3 months after surgery; Group III ( $\mathrm{n}=80$ ) prior to laparoscopic removal of the ovarian cyst used gonadotropin-releasing hormone agonists - Triptorelin 3.75 mg intramuscularly for 2 months, as well as three months after surgery. The control group consisted of 30 healthy women of reproductive age with regular menstrual periods. All patients underwent transvaginal ultrasound, counting the number of antral follicles before and after treatment. Serum hormone levels (FSH, prolactin, thyrotropic hormone, anti-Mullerian hormone, inhibin B) were determined by enzyme-linked immunosorbent assay on Cobas e-411 analyzer (Roche Diagnostics, Switzerland) on day 2-3 of the menstrual cycle and on day 2-3 of the first menstrual period after the end of treatment. Laparoscopic removal of the cyst was performed with exfoliation of the cyst, hemostasis on the wound surface of the bed of the cyst was performed with a bipolar electrocoagulator. Bipolar coagulation and resection of the ovarian tissue with no potential was used during surgical treatment of the ovaries, which made it possible to preserve the intact portion of the ovary as much as possible.

Results: Analysis of ovarian reserve indices, namely number of antral foliculs, number of antral follicles, AMG, and inhibin B levels in all examined patients with ovarian endometriomas were significantly lower than those of the control group before the start of treatment: in the ovarian endometrial group group 1.26 times $(p<0.01)$, inhibin $B$ -1.5 times $(p<0.01)$, the number of antral follicles -1.2 times $(p<0.01)$, due to the development dystrophic changes of the follicular apparatus due to prolonged compression, hypoxia, fibrosis in the ovaries.

Patients who planned pregnancy were advised to have an active sexual life before menstruation was restored. In 23 (46.9\%) of 49 patients who had reproductive plans, pregnancy occurred without first menstruation after a course of gonadotropin-releasing hormone agonists, 12 (24.5\%) women became pregnant during the first three menstrual cycles. Extracorporeal fertilization was recommended for women who did not have pregnancy within 6 months of surgery. For two years in women who did not plan pregnancy, recurrence of endometriosis was not observed.

Conclusions: The combination of laparoscopic treatment with gonadotropin-releasing hormone agonists in patients with endometriosis with infertility allowed to restore reproductive function in $71.4 \%$ of women, which indicates the effectiveness of the treatment method used. In addition, it helps to achieve lasting remission and addresses the socio-social problems of women's health and maternity.
\end{abstract}

KEY WORDS: infertility, ovarian endometrioma, agonists of gonadotropin-releasing hormone, ovarian reserve

\section{INTRODUCTION}

Genital endometriosis is one of the leading causes of impaired quality of life and impaired fertility in the female population. According to Adamson et al. (2010), approximately 176 million women (6 to $10 \%$ of the female population) of reproductive age worldwide suffer from certain symptoms of this disease. Endometriosis is the cause of pelvic pain in 50-60\% of adolescent girls and women of different age groups, as well as $50 \%$ of infertility cases in women of reproductive age $[1,2]$. Currently, endometriosis is third in the structure of diseases of the reproductive organs and remains an important medical and social problem. In recent years, there has been a tendency to increase the incidence of endometriosis in young women who have not given birth. Endometriosis leads to func- tional and structural changes in the reproductive system, often negatively affecting the emotional state of women, significantly reducing the quality of life.

According to modern scientific data, the main mechanism of endometriosis is the violation of the ratio of hormonal and immune balance in the body of a woman $[3,4,5]$. According to current ideas about the nature of endometriosis, this disease should be regarded as a pathological process with a chronic, recurrent course. Endometriosis is formed and develops against the background of impaired immune, molecular genetic and hormonal relationships in the female body. Therefore, the main mechanism of endometriosis is the violation of the hormonal and immune balance in a woman's body [6,7]. First of all, the peculiarities of activity of the hypothalamic-pituitary-ovarian system are important for 
the occurrence of endometriosis. In patients with endometriosis there are chaotic peak emissions of follicle-stimulating (FSH) and luteinizing ( $\mathrm{LH})$ hormones, there is a decrease in the basal level of progesterone, many have found hyperprolactinemia and impaired androgenic function of the adrenal cortex. Despite this, patients with genital endometriosis note a high incidence of ovarian luteinization syndrome, while maintaining the external parameters of the ovulatory menstrual cycle (biphasic basal temperature, sufficient level of progesterone in the middle of the lutein, secretion. The examination of patients with endometriosis revealed local morphological changes of the ovaries, especially in the defeat of the ovaries themselves. Ovarian endometriomas are observed in $17-44 \%$ of the total number of patients suffering from endometriosis $[8,9,10]$.

About 30-40\% of women with endometriosis suffer from infertility $[11,12]$. The problem of endometriosis and infertility remains relevant in modern gynecology. Endometriosis is diagnosed late, from the moment of onset of the disease until diagnosis 7-8 years. The chronic course of endometriosis and its high potential for recurrence after various types of treatment determine the relevance of indepth studies of pathogenesis and improvement of therapeutic approaches to this disease $[13,14]$. Patients with endometriosis-associated infertility are the most difficult contingent to treat for endometriosis. In this situation, the main problematic issues in the treatment of endometriosis are not sufficiently high efficacy with regard to long-term relapse prevention, both individually and in combination with surgery; antireproductive effect of the most effective groups of drugs (agonists of gonadotropin-releasing hormones, hormonal contraceptives, gestagens, antiestrogens, aromatase inhibitors).

Modern approaches to the treatment of endometriosis are to combine pathogenetically sound conservative methods aimed at suppressing estrogenic stimulation of endometrioid heterotopias, inflammatory mediators and immunotherapy with surgical methods of treatment, namely, ovarian endometrial removal by laparoscopic method and rehabilitation therapy after surgery $[11,14]$.

The purpose of surgical treatment is to maintain and restore childbearing function in women with endometriosis. However, after surgical treatment, the recurrence rate is high: in $15 \%$ of cases - one year after surgery, in $25 \%$ - after 4 years. Medicinal (hormonal) treatment at small sizes of endometriotic cysts allows to keep an ovarian reserve, but does not lead to increase of fertility at women with large forms of endometriosis, and operative treatment of large forms of endometriosis with hormonal correction after, can provide prevention of recurrence prevention. Laparoscopic endometrial leaching is the method of choice in conservative treatment and is considered better than fenestration and ablation of the capsule due to decreased pain, recurrence rates, and an increase in the number of spontaneous pregnancies [8]. When performing endometrial surgery in women of reproductive age, maintaining the ovarian reserve is very important. It is defined as the functional potential of the ovary and reflects the amount and quality of follicles remaining in the ovaries. Currently, tests showing the ovarian reserve are serum antimuller hormone (AMG) levels, a count of 2-3 days of the menstrual cycle of the number of antral follicles (AFC), determination of serum follicle stimulating hormone levels and 12 inhibin. The level of anti-Mullerian hormone does not depend on the day of the menstrual cycle and on the use of gonadotropin-releasing hormone agonists or oral contraceptives $[12,14]$. Recent studies have demonstrated that laparoscopic endometrial leaching is associated with a decrease in ovarian reserve due to excessive removal of ovarian tissue and hemostasis using bipolar coagulation of ovarian tissues (thermal damage) or suture (local tissue injury)]. The results of histological examinations of the removed endometrium have shown the unwanted removal of a portion of healthy ovarian tissues, along with the endometrioid tissue, especially in dangerous areas close to the ovarian gates. At the same time, other studies have shown that laparoscopic cystectomy in endometrioid cysts using highly skilled surgical techniques does not lead to significant damage to the ovarian reserve [6].

\section{THE AIM}

The aim of the study was to improve the results of treatment in patients with endometriosis by using a combination method of therapy.

\section{MATERIALS AND METHODS}

The study was conducted at the Sumy Regional Clinical Perinatal Center and the Department of Obstetrics and Gynecology of Sumy State University during 2016-2018. For two years, 136 women of reproductive age underwent laparoscopic access for ovarian endometrium were monitored: Group I $(\mathrm{n}=24)$ did not receive any hormonal treatment in the perioperative period; Group II $(\mathrm{n}=32)$ - received gonadotropin-releasing hormone agonists within 3 months after surgery; Group III $(\mathrm{n}=80)$ prior to laparoscopic removal of the ovarian cyst used gonadotropin-releasing hormone agonists - Triptorelin $3.75 \mathrm{mg}$ intramuscularly for 2 months, as well as three months after surgery. The control group consisted of 30 healthy women of reproductive age with regular menstrual periods. Exclusion criteria were pregnancy, diabetes and other endocrine, systemic diseases, somatic pathology in the stage of decompensation, body mass index above $30 \mathrm{~kg} / \mathrm{m}^{2}$, malignancies, postoperative pathologic exclusion of endometrioid cystogenesis, combination of endometriosis and ovarian scleropolistosis.

All patients were examined according to existing clinical guidelines regarding the algorithm of clinical and laboratory monitoring in the preoperative period. All patients underwent transvaginal ultrasound, counting the number of antral follicles before and after treatment. Serum hormone levels (FSH, prolactin, thyrotropic hormone, anti-Mullerian hormone, inhibin B) were determined by enzyme-linked immunosorbent assay on Cobas e-411 analyzer (Roche Diagnostics, Switzerland) on day 2-3 of the menstrual cycle 
and on day 2-3 of the first menstrual period after the end of treatment. For dynamic monitoring of the condition of women, ultrasound examination of the pelvic organs and vaginal examination after 1 and 4 months.

Laparoscopic removal of the cyst was performed by exfoliation, hemostasis of the wound surface of the bed of the cyst was performed with a bipolar electrocoagulator. Bipolar coagulation and resection of the ovarian tissue without energy was used during surgical treatment of the ovaries, which made it possible to preserve the intact portion of the ovary as much as possible. All the removed tissues were subjected to histological examination. A menstrual response was observed after surgery on days $1-3$. On day $3-5$, this reaction was prescribed treatment with gonadotropin-releasing hormone agonists: Triptorelin $3.75 \mathrm{mg}$ intramuscularly. Triptorelin is a synthetic analogue of the naturally occurring gonadotropin-releasing hormone with a similar structure. The course of treatment was administered 3-5 injections once every 28 days. In satisfactory condition, women were recommended on days 3-4.

Statistical processing and analysis of the obtained data was performed using «Microsoft Excel» programs using the methods of mathematical statistics and the software package Statistica 8.0. Charts and diagrams were built using «Microsoft Excel». Mathematical processing of indicators was performed using the methods of variation statistics for comparing inhaled sets by averages using the $t$-Student test.

This study was approved by the ethics committee of the Sumy State University, Ukraine. All procedures were carried out in accordance with the ethical standards of the responsible committee on human experimentation and with the Helsinki Declaration of 1975, as revised in 2000.

\section{RESULTS}

The age of the patients in group I was $(27.46 \pm 0.42)$ years, in group II - (27.37 \pm 0.35$)$ years, in group III - (27.84 \pm $0.46)$ years, in the control group - $(27.13 \pm 0.64)$, so no significant difference was found. The groups were also homogeneous according to anthropometry, gynecological, reproductive, somatic, infectious history.

The structure of gynecological morbidity among the surveyed was as follows: inflammatory diseases of the uterus and appendages - in 87 (64\%), menstrual disorders - in 35 (25.7\%), infertility - in 76 (56\%). In addition, patients complained of constant pain, which increased on the eve and during menstruation, in sexual intercourse, with irradiation in the lumbar region, sacrum, rectum; dysuric phenomena and gastrointestinal disorders. In 11 (8\%) women the course of the disease was asymptomatic, the ovarian cyst was diagnosed during the prophylactic examination.

The sizes of ovarian endometriums did not differ in the examined groups. Thus, the average size of the endometrium in group I was $4.34 \pm 0.24 \mathrm{~cm}$; in group II -4.91 $\pm 0.18 \mathrm{~cm}$; in group III $-4.71 \pm 0.16 \mathrm{~cm}$. It should be noted that in patients with bilateral ovarian endometri- oma, unilateral cyst larger than $6 \mathrm{~cm}$, the CA-125 level exceeds $35 \mathrm{nmol} / \mathrm{l}$ and even up to $60 \mathrm{nmol} / \mathrm{l}$. These patients are sure to have been consulted and examined by an oncologist. The main indications for laparoscopic treatment of patients with ovarian endometriosis were: unilateral endometrioma - in $76(56 \%)$ and bilateral - in $60(44 \%)$, their combination. With infertility occurred - in $76(56 \%)$ patients. In the ratio of the number of unilateral and bilateral endometriums, all examined groups did not differ. During the endoscopic diagnosis and treatment, "typical" signs of the endometrioma were observed: ovarian formation with a dense capsule of dark blue hue and fragments of blue-purple color - in 50\% (fig 1); black, cyanotic, dark red patches on the peritoneum surface - 36\%; scar tissue surrounding endometrioid heterotopia - 23\%; "Atypical" hypervascular zones - in $53 \%$; pericardial fusion $-46 \%$; yellow-brown spots on the peritoneum surface $-36 \%$; petechial peritoneum $30 \%$; glandular tumors on the surface of the peritoneum - in 16\%; areas of white opaque peritoneum - $10 \%$ and circular peritoneum defects $-6 \%$.

Analysis of ovarian reserve indices, namely number of antral foliculs, number of antral follicles, AMG, and inhibin $B$ levels in all examined patients with ovarian endometriomas were significantly lower than those of the control group before the start of treatment: in the ovarian endometrial group group 1.26 times $(\mathrm{p}<0.01)$, inhibin $\mathrm{B}-1.5$ times ( $\mathrm{p}$ $<0.01)$, the number of antral follicles -1.2 times $(p<0.01)$, due to the development dystrophic changes of the follicular apparatus due to prolonged compression, hypoxia, fibrosis in the ovaries (fig 2).

The results of the study of indicators of ovarian reserve in the examined patients of reproductive age with ovarian endometriomas before and after treatment are presented in Table I.

\section{DISCUSSION}

At the end of treatment, no significant difference was observed between group I and group II indicators, but group III showed significantly better AMH levels, a greater number of antral foliculs antral follicles, and Inhibin-B levels due to preoperative use of gonadotropin-rilon agonists, which reduce the blood supply to the cyst capsule, reduce the proliferative activity of the endometriosis, reduce the intensity and duration of coagulation hemostasis of the wound surface of the cyst bed.

The menstrual cycle in all women receiving gonadotropin-releasing hormone agonists occurred 3-4 months after the last injection.

Patients who planned pregnancy were advised to have an active sexual life before menstruation was restored. In 23 (46.9\%) of 49 patients who had reproductive plans, pregnancy occurred without first menstruation after a course of gonadotropin-releasing hormone agonists, 12 (24.5\%) women became pregnant during the first three menstrual cycles. Extracorporeal fertilization was recommended for women who did not have pregnancy within 6 months 


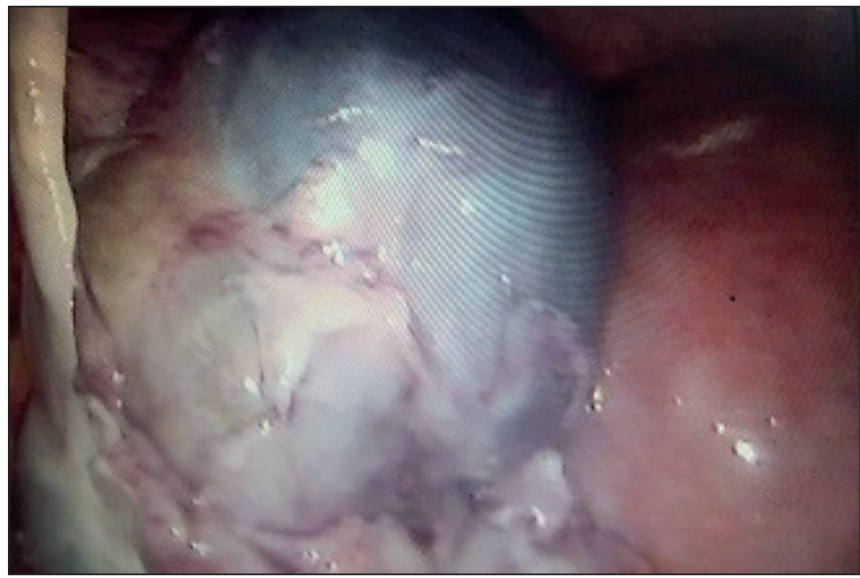

Fig. 1. Ovarian formation with a dense capsule of dark blue hue and fragments of blue-purple color.

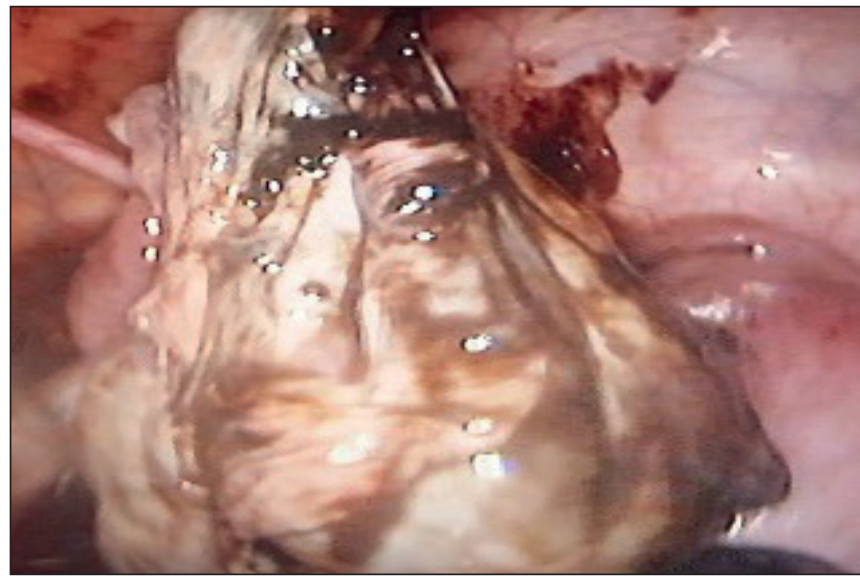

Fig. 2. Development dystrophic changes of the follicular apparatus due to prolonged compression, hypoxia, fibrosis in the ovaries

Table I. The results of the study of indicators of ovarian reserve in the examined patients of reproductive age with ovarian endometriomas before and after treatment, $\mathrm{t}$

\begin{tabular}{|c|c|c|c|c|c|}
\hline Index & Time of treatment & I group & II group & III group & Control \\
\hline \multirow{2}{*}{$\mathrm{AMH}, \mathrm{ng} / \mathrm{ml}$} & before of treatment & $1,44 \pm 0,04^{*}$ & $1,46 \pm 0,03^{*}$ & $1,40 \pm 0,04^{*}$ & \multirow{2}{*}{$1,76 \pm 0,04$} \\
\hline & afther of of treatment & $1,07 \pm 0,04^{*}$ & $1,12 \pm 0,03^{*}$ & $1,27 \pm 0,04^{*} / * *$ & \\
\hline \multirow{2}{*}{ NAF } & before of treatment & $3,93 \pm 0,14^{*}$ & $4,00 \pm 0,11^{*}$ & $4,01 \pm 0,12^{*}$ & \multirow{2}{*}{$5,49 \pm 0,25$} \\
\hline & afther of treatment & $4,48 \pm 0,14^{*}$ & $4,46 \pm 0,13^{*}$ & $4,89 \pm 0,12^{*} / * *$ & \\
\hline \multirow{2}{*}{$\mathrm{FSH}, \mathrm{Cl} / \mathrm{I}$} & before of treatment & $3,88 \pm 0,17$ & $3,71 \pm 0,11$ & $3,66 \pm 0,14$ & \multirow{2}{*}{$4,02 \pm 0,18$} \\
\hline & afther of treatment & $4,23 \pm 0,16$ & $4,26 \pm 0,19$ & $4,17 \pm 0,17$ & \\
\hline \multirow{2}{*}{ Inhibin-B, ng/l } & before of treatment & $29,79 \pm 0,60^{*}$ & $29,32 \pm 0,39^{*}$ & $29,67 \pm 0,49^{*}$ & \multirow{2}{*}{$42,72 \pm 1,00$} \\
\hline & afther of treatment & $31,73 \pm 0,85^{*}$ & $32,29 \pm 0,62^{*}$ & $37,61 \pm 0,95 * / * *$ & \\
\hline
\end{tabular}

Note. ${ }^{*}$ - reliability of the difference, $p<0.05$.

of surgery. For two years in women who did not plan pregnancy, recurrence of endometriosis was not observed.

\section{CONCLUSIONS}

1. Persistence of endometrium in the ovaries of women of reproductive age leads to a decrease in ovarian reserve.

2. The use of combined treatment of patients with ovarian endometriosis contributes to the normalization of hormonal function of the hypothalamic-pituitary-ovarian system.

3. Post-operative administration of gonadotropin-releasing hormone agonists does not affect ovarian reserve indices in women with endometriomas, whereas pre-operative use in combination with postoperative surgery contributes to the reduction of ovarian reserve damage after surgical treatment.

4. The combination of laparoscopic treatment with gonadotropin-releasing hormone agonists in patients with endometriosis with infertility allowed to restore reproductive function in $71.4 \%$ of women, which indicates the effectiveness of the treatment method used. In addition, it helps to achieve lasting remission and addresses the socio-social problems of women's health and maternity.

\section{REFERENCES}

1. Koval H.D., Chopyak V.V., Kamyshnyi O.M. Transcription regulatory factor expression in T-helper cell differentiation pathway in eutopic endometrial tissue samples of women with endometriosis associated with infertility. Cent Eur J Immunol. 2018; 43(1): 90-96.

2. Hehenkamp W.J., Looman C.W., Themmen A.P. et al. Anti-Mullerian hormone levels in the spontaneous menstrual cycle do not show substantial fluctuation. J. Clin. Endocrinol. Metab. 2006; 91(10): 4057-63. doi: 10.1210/jc.2006-0331.

3. de Ziegler D., Borghese B., Chapron C. Endometriosis and infertility: pathophysiology and management. Lancet. 2010; 376(9742):730-38. doi: 10.1016/S0140-6736(10)60490-4.11

4. Yang X.H., Ji F., Aili A. et al. Effects of laparoscopic ovarian endometriosis cystectomy combined with postoperative GnRHa therapy on ovarian reserve, pregnancy, and outcome recurrence. Clin. Exp. Obstet. Gynecol. 2014; 41(3): 272-275.

5. Hachisuga T. Histopathological analysis of laparoscopically treated ovarian endometriotic cysts with special reference to loss of follicles. Hum. Reprod. 2002; 17(2); 432-435.

6. Bhat R.G., Dhulked S., Ramachandran A. et al. Laparoscopic cystectomy of endometrioma: Good surgical technique does not adversely affect ovarian reserve. J. Hum. Reprod. Sci. 2014; 7(2): 125-129. doi: 10.4103/0974-1208.138871. 
7. Ebert A.D., Hollauer A., Fuhr N. et al. Laparoscopic ovarian cystectomy without bipolar coagulation or sutures using a gelantinethrombin matrix sealant (FloSeal): first support of a promising technique. Arch. Gynecol. Obstet. 2009; 280 (1):161-165.

8. Hobday C.D., Milam M.R., Milam R.A. et al. Postoperative small bowel obstruction associated with use of hemostatic agents. J. Minim. Invasive Gynecol. 2009; 16(2): 224-226.

9. Deb S., Campbell B.K., Pincott-Allen C. et al. Quantifying effect of combined oral contraceptive pill on functional ovarian reserve as measured by serum anti-Mullerian hormone and small antral follicle count using three-dimensional ultrasound. Ultrasound Obstet Gynecol. 2012; 39(5): 574-580. doi: 10.1002/uog.10114.

10. Benaglia L., Somigliana E., Vighi V. et al. Rate of severe ovarian damage following surgery for endometriomas. Hum. Reprod. 2010; 25(3):678-682.

11. La Marca A., Stabile G., Artenisio A.C., Volpe A. Serum anti-Mullerian hormone throughout the human menstrual cycle. Hum. Reprod. 2006; 21(12): 3103-3107. doi: 10.1093/humrep/del291.

12. Pados G., Tsolakidis D., Assimakopoulos E. et al. Sonographic changes after laparoscopic cystectomy compared with three-stage management in patients with ovarian endometriomas: a prospective randomized study. Hum. Reprod. 2010; 25(3): 672-677.

13. Kristensen S.L., Ramlau-Hansen C.H., Andersen C.Y. et al. The association between circulating levels of antimullerian hormone and follicle number, androgens, and menstrual cycle characteristics in young women. Fertil. Steril. 2012; 97(3): 779-785. doi: 10.1016/j. fertnstert.2011.12.017.

14. Muzii L., Di Tucci C., Di Feliciantonio M. et al. The effect of surgery for endometrioma on ovarian reserve evaluated by antral follicle count: a systematic review and meta-analysis. Hum. Reprod. 2014; 29(10): 2190-2198. doi: 10.1093/humrep/deu199.
The work is carried out within the framework of the research work "Optimization of diagnosis and prevention of diseases of the reproductive system and development of pathogenically grounded methods for their correction" (state registration number 011U001801).

\section{ORCID and contributorship:}

Iryna M. Nikitina - 0000-0001-6595-2502 A,E,F

Volodymyr I. Boiko - 0000-0001-6069-4268 ${ }^{\mathrm{C}}$

Svitlana A. Smiian - 0000-0002-7679-2302 ${ }^{B, D}$

Tetiana V. Babar - 0000-0002-5889-2429 ${ }^{\mathrm{B}}$

Natalia V. Kalashnyk - 0000-0003-1515-9105 ${ }^{B}$

Alesia V. Boiko - 0000-0002-6994-7564 ${ }^{\mathrm{F}}$

Natalia A. Ikonopystseva - 0000-0002-8668-724X ${ }^{F}$

Maryna A. Bolotna - 0000-0003-1982-9022 ${ }^{D}$

\section{Conflict of interest:}

The Authors declare no conflict of interest.

\section{CORRESPONDING AUTHOR \\ Iryna M. Nikitina}

Department of Obstetrics and Gynecology, Sumy State University

12, Rymskogo-Korsakova st., 40007 Sumy, Ukraine

tel:+380662947360

e-mail:nikitina1med@gmail.com

Received: 22.01 .2020

Accepted: 30.03 .2020

A - Work concept and design, B - Data collection and analysis, C - Responsibility for statistical analysis,

$\mathbf{D}$-Writing the article, $\mathbf{E}$-Critical review, $\mathbf{F}$ - Final approval of the article 